\title{
Unconstrained Body Movement
}

National Cancer Institute

\section{Source}

National Cancer Institute. Unconstrained Body Movement. NCI Thesaurus. Code C90480.

The ability to move body parts and limbs without physical restriction. 